\title{
INFLUENCE OF PRIOR AND VISUAL INFORMATION ON EYE MOVEMENTS IN AMBLYOPIC CHILDREN
}

Coralie Hemptinne ${ }^{a, b}$ MD, Nicolas Deravet ${ }^{a, c}$ MS PhD, Jean-Jacques Orban de Xivryd,e MS PhD, Philippe Lefèvre ${ }^{a, c}$ MS PhD, Demet Yüksela,b MD PhD

a Institute of Neuroscience, Université catholique de Louvain, Louvain-La-Neuve, Belgium

b Ophthalmology Department, Cliniques Universitaires Saint-Luc, Brussels, Belgium

c Institute of Information and Communication Technologies, Electronics, and Applied Mathematics, Louvain-La-Neuve, Belgium

d Department of Movement Sciences, Movement Control and Neuroplasticity Research Group, Katholieke Universiteit Leuven, Leuven, Belgium

e Leuven Brain Institute, Katholieke Universiteit Leuven, Leuven, Belgium

Corresponding author:

Coralie Hemptinne

Ophthalmology Department, Cliniques Universitaires Saint-Luc

Avenue Hippocrate 10, 1200 Brussels, Belgium

coralie.hemptinne@uclouvain.be

Phone number: +32 478663699

Fax number: +32 27642988

ORCID identifier: 0000-0003-0717-3804 


\section{ABSTRACT}

This study analyzed the characteristics of pursuit and assessed the influence of prior and visual information on eye velocity and saccades in amblyopic and control children, in comparison to adults. Eye movements of 41 children ( 21 amblyopes and 20 controls) were compared to eye movements of 55 adults (18 amblyopes and 37 controls). Participants were asked to pursue a target moving at a constant velocity. The target was either a 'standard' target, with a uniform color intensity, or a 'noisy' target, with blurry edges, to mimic the blurriness of an amblyopic eye. Analysis of pursuit patterns showed that the onset was delayed, and the gain was decreased in control children with a noisy target in comparison to amblyopic or control children with a standard target. Furthermore, a significant effect of prior and visual information on pursuit velocity and saccades was found across all participants. Moreover, the modulation of the effect of visual information on the pursuit velocity by group, that is amblyopes or controls with a standard target, and controls with a noisy target, was more limited in children. In other words, the effect of visual information was higher in control adults with a standard target compared to control children with the same target. However, in the case of a blurry target, either in control participants with a noisy target or in amblyopic participants with a standard target, the effect of visual information was larger in children.

\section{KEYWORDS}

Child, Adult, Amblyopia, Smooth pursuit, Saccade, Short-term memory

\section{DECLARATIONS}

\section{FUNDING}

Funding information is not applicable. No funding was received.

\section{CONFLICTS OF INTEREST / COMPETING INTERESTS}

The authors declare that they have no conflict of interest.

\section{AVAILABILITY OF DATA AND MATERIAL}

With the exception of confidential patient information, relevant data can be sent upon request.

\section{CODE AVAILABILITY}

Program code and model parameter data sets are freely available for academic use. 


\section{INTRODUCTION}

Humans visually interact with their environment using smooth pursuit and saccades (Orban De Xivry and Lefèvre 2007). Saccades and smooth pursuit are programmed in the brain taking into account sensory visual inputs and predictions based on a short-term memory of prior visual information (Deravet et al. 2018). The models that integrate these two inputs include the Bayesian integration and the Kalman filtering, with weights linked to these two inputs' reliability (Orban de Xivry et al. 2013). Given their lower ability to estimate the position, velocity and acceleration of objects, children preferentially rely on visual feedback to achieve motor tasks (Rösblad 1996).

At birth, the visual system is not as mature as it is in adults, unlike other sensory systems such as the auditory system. The saccadic system becomes comparable in children and adults at about 6 years (Ego et al. 2013). Saccadic peak velocity is equal to or slightly higher in children than in adults, with a similar saccade accuracy (Accardo et al. 1995; Irving et al. 2006; Salman, Sharpe, Eizenman, et al. 2006). However, saccade latency decreases with age in children until 12-15 years of age (Fukushima et al. 2000; Irving et al. 2006; Luna et al. 2004). Higher saccade latencies are associated with more accurate saccades, that is a smaller position error after saccades.

The pursuit system continuously matures until late adolescence (Rütsche et al. 2006; Salman, Sharpe, Lillakas, et al. 2006; Von Hofsten and Rosander 1997). In the pursuit of predictable stimuli, the pursuit gain, defined as the ratio of the eye to the target velocity, increases until between 13 and 18 years of age (Accardo et al. 1995; Katsanis et al. 1998; Salman, Sharpe, Lillakas, et al. 2006), or until 6-7 years for lower target velocities (Ross et al. 1993). For instance, in the pursuit of a sinusoidally moving target, pursuit gain increased from 0.7 at 8 years old to 1.0 at 19 years old (Salman, Sharpe, Lillakas, et al. 2006). In the pursuit of unpredictable targets at constant velocity, adults react sooner and accelerate faster than children during the initiation of pursuit (Ego et al. 2013), with acceleration increasing until 14-16 years old, average pursuit latency decreasing until 8-9 years old, and latency standard deviation decreasing until 14-16 years old.

While the development of pursuit and saccades in children has been analyzed by many authors, the influence of prior information on the oculomotor behavior of children has been scarcely studied. In addition, although the oculomotor performance in amblyopia, a pathology associated with motion perception deficit, has been studied in adults, little is known regarding the characteristics of pursuit and saccades in amblyopic children.

The present study aimed to analyze the characteristics of pursuit and to assess the influence of prior and visual information on eye velocity and on saccades for children compared to adults in three subgroups: control with standard target, control with noisy target and amblyopic with standard target.

\section{METHOD}

\section{PARTICIPANTS}

The research protocol followed the tenets of the Declaration of Helsinki and was approved by the University of Louvain's Human Biomedical Ethics Board. 59 volunteers between 5 and 13 years old equally distributed in four age categories, including 30 children who were treated for amblyopia at the Cliniques Universitaires Saint-Luc and 29 age-matched healthy controls without strabismus nor amblyopia, were tested between March 2015 and December 2019. Their results were compared to 58 adolescents and adults (referred to as "adults" in the remainder of the manuscript) between 12 and 61 years old, including 20 amblyopic patients and 38 controls. All participants and, in the case of children, their carers provided informed written consent before participating in the study. 
Inclusion criteria for all participants included the following: the patient (1) has completed the full protocol; (2) understands the instructions; (3) has given, together with their carer, informed written consent (4) presents no general health disease, has a normal neurologic status and does not take any medication.

Furthermore, amblyopic patients were required to have unilateral amblyopia with a visual acuity lower than 0.15 logMAR. Lastly, controls were required to have no ophthalmological disease particularly strabismus. All participants had normal binocular vision, and corrected visual acuities of 0 logMAR or better in both eyes.

The exclusion criteria included several conditions regarding the quality of the recorded data (see the Data processing section).

\section{EXPERIMENTAL SET-UP AND STIMULI}

Participants were seated on a chair in a darkened room and were asked to fixate on a colored target projected by a cine8 Barco projector (Barco Inc., Kortrijk, Belgium) at a refresh rate of $100 \mathrm{~Hz}$. At a viewing distance of $150 \mathrm{~cm}$, the screen subtended $65^{\circ}$ (horizontal) $\times 50^{\circ}$ (vertical) of visual angle. Head position was kept stable using a forehead and chin rest. Eye movements of the non-dominant eye were recorded at $1000 \mathrm{~Hz}$ with an Eyelink 1000 (SR Research, Ottawa, Ontario, Canada), with the other eye occluded.

The experiment started with a calibration sequence, during which participants were asked to fixate on a target initially located in the center of the screen. The target would then move to various points on the screen (e.g., right, left, up or down). This sequence was repeated every 30 trials, and a break was proposed to participants before each calibration sequence. The calibration sequence was followed by stimulus blocks. These blocks included training and test trials. In the trials of a given stimulus block, participants were asked to fixate a target appearing at the center of the screen for $500 \mathrm{~ms}$ and then to pursue the target moving at a constant velocity $\left(15^{\circ} / \mathrm{s}\right.$ or $\left.20^{\circ} / \mathrm{s}\right)$ in a given direction $\left(-20^{\circ}, 0^{\circ}, 20^{\circ}, 160^{\circ}\right.$, $180^{\circ}$ or $\left.200^{\circ}\right)$. In the following stimulus block, the target moved in a different direction, which remained constant throughout the block. Each stimulus block included 1 to 4 training trials and, in $80 \%$ of the blocks, a test trial. Training trials were defined as trials with a constant direction and target velocity, in order to build a short-term memory of prior visual information. In the test trial, the direction remained identical, however, for $80 \%$ of the blocks, the velocity changed by $\pm 5 \%$ s (Fig. 1 b). The experiment lasted approximately 30 minutes.

Fig. 1 (a) Example of two stimulus blocks, including one or several training trials at a given velocity and one test trial at a different velocity (b) Structure of blocks

For a given experiment, the target was either a 'standard' target, which consisted of a red disk with a diameter of $0.8^{\circ}$ and a uniform color intensity, or a 'noisy' target, which was a red disk with the same luminance, but with blurry edges, according to a Gaussian distribution $\left(\sigma=1.27^{\circ}\right)$. The noisy target was defined and implemented in controls to degrade the quality of the visual information (Deravet et al. 2018, 2019). Since bad visual acuity is one of the core deficits in amblyopia, a noisy target simulating blurriness is a way to mimic the abnormal visual perception of an amblyopic eye in a global manner. However, we are aware that blurriness is not a complete representation of the range and breadth of visual deficits seen in amblyopia. Nonetheless, modeling these different patterns of visual deficits is beyond the scope of this paper. It would certainly be an opening for further studies.

Controls completed two conditions, first with a noisy target then with a standard target. Amblyopic patients only completed one condition with a standard target. The adults and children were subdivided into 3 groups: standard (control subjects with a standard target), noisy (control subjects with a blurred target), and amblyopic (amblyopes with a standard target).

\section{DATA PROCESSING}


Missing data in the Eyelink output were considered to be blinks and removed from the data, including a margin preceding and following the blink. The resulting data was low-pass filtered at $35 \mathrm{~Hz}$ in Matlab. Eye velocity and acceleration were evaluated based on the position signal using a central difference algorithm. Saccades were detected by means of a $500 \% \mathrm{~s}^{2}$ acceleration threshold and replaced by a linear interpolation.

Pursuit onset was defined as the intersection of two regression lines approximating, respectively, low velocity (anticipatory) eye movements as a plateau and the subsequent (visually-guided) acceleration as a ramp, within the interval $[-100,300] \mathrm{ms}$. This estimation was considered as valid if no blink occurred in the $50 \mathrm{~ms}$ following the computed pursuit onset, and if eye velocity during the initial plateau remained lower than $4 \%$ s.

Steady-state eye velocity was considered to be the slope of a regression line approximating eye position for an interval of at least $70 \mathrm{~ms}$ starting at the first catch-up saccade and ending at the following saccade. If there was no such interval, smooth eye displacement during saccades was approximated by the integral of the linear interpolation of the saccades' eye velocity data, and included in the fit.

Position error was estimated $100 \mathrm{~ms}$ before saccade onset, at the time when it was assumed to be assessed by participants (Becker and Jürgens 1979). It was defined as the difference between target and eye position.

The accuracy of saccades was evaluated by computing the saccade residuals, that is the difference between actual and ideal saccade amplitudes. Ideal saccade amplitudes were assumed to be the difference between target position at saccade offset and eye position at saccade onset. These residuals were determined for trials 2 to 4 , as in trial 1 pursuit onset occurred later, with larger saccades.

Trials were considered as abnormal and removed from the data if: (1) the eye position error was greater than $5^{\circ}$ in the last $100 \mathrm{~ms}$ of fixation; (2) there was 90 or more missing data considered to be blinks in the 450 ms of pursuit; (3) eye displacement was smaller than $15 \%$ of target displacement; (4) position error during pursuit was greater than $8^{\circ}$ for more than $100 \mathrm{~ms}$; (5) participants performed a saccade greater than $25^{\circ}$ or a reverse saccade; (6) the steady-state pursuit gain was smaller than 0.2 ; (7) less than $5 \%$ of the target displacement was associated with smooth pursuit; (8) pursuit started less than $50 \mathrm{~ms}$ after the start of target displacement. In addition, if more than $60 \%$ of a participant's recorded data was rejected, that participant was excluded from the study.

\section{EVALUATION OF PRIOR AND VISUAL INFORMATION EFFECTS}

In order to estimate the effect of prior information, we compared the pursuit characteristics of a test trial and a training trial with the same trial number and target velocity but preceded by trials with different target velocity. For instance, we compared the pursuit gain of:

- Test trial \#2, with a target velocity of $15 \mathrm{deg} / \mathrm{s}$ and preceded by training trial \#1 at $20 \mathrm{deg} / \mathrm{s}$,

- And training trial \#2 at $15 \mathrm{deg} / \mathrm{s}$ (identical visual input) and preceded by training trial \#1 at 15 $\mathrm{deg} / \mathrm{s}$ (different prior information).

As the visual input was identical for the compared trials, eye movement differences between them were assumed to derive from the different prior visual information. The extent to which prior visual information influenced eye velocity was assessed using the following normalized ratio (Deravet et al. 2018):

mean (eye velocity of training trial) - eye velocity of test trial

$\overline{\text { prior target velocity of training trial - prior target velocity of test trial }}$ 
Similarly, the effect of visual input was evaluated by comparing the pursuit characteristics of a test trial and a training trial with the same trial number and preceded by trials with the same target velocity, but with different target velocity. For instance, we compared the pursuit gain of:

- Test trial \#3, with a target velocity of $10 \mathrm{deg} / \mathrm{s}$ and preceded by training trial \#2 at $15 \mathrm{deg} / \mathrm{s}$,

- And training trial \#3 at $15 \mathrm{deg} / \mathrm{s}$ (different visual input) and preceded by training trial \#2 at $15 \mathrm{deg} / \mathrm{s}$ (identical prior visual information).

As the prior visual information was identical for the compared trials, eye movement differences between them were assumed to derive from the different visual inputs. The extent to which visual input influenced eye velocity was assessed using the following normalized ratio (Deravet et al. 2018):

$$
\frac{\text { mean (eye velocity of training trial) - eye velocity of test trial }}{\text { target velocity of training trial - target velocity of test trial }}
$$

\section{STATISTICAL ANALYSES}

Hypothesis tests were performed using type III ANOVA, with the evaluation of the denominator degrees of freedom performed using the Kenward-Roger method. To analyze the pursuit characteristics (pursuit onset and steady-state pursuit gain), we used the following factors: age (adults vs. children), group (control participant with standard target, control participant with noisy target and amblyopic participant with standard target) and target velocity (15 deg/s vs. $20 \mathrm{deg} / \mathrm{s}$ ). The nested structure of the data was taken into account by including random intercepts for participants into the models for all relevant analyses.

To analyze the effect of prior information on the smooth pursuit response and on the amplitude of catch-up saccades, we used the normalized effect of prior information (see above) as dependent variable and the following factors: age, group, target velocity and prior information (no prior information vs. prior information). In this case, a main effect of the factor "prior" would indicate that prior information influences the oculomotor response.

To analyze the effect of visual information on the smooth pursuit response and on the amplitude of catch-up saccades, we performed a first analysis on the raw oculomotor data (pursuit velocity or saccade amplitude) to demonstrate the presence of an effect and then a second analysis with the normalized effect of visual information (see above) as dependent variable. In the first analysis, we used the following factors: age, group, target velocity and visual information $(-5 \mathrm{deg} / \mathrm{s},+5 \mathrm{deg} / \mathrm{s}$ or no change with respect to previous trial). In this case, a main effect of the factor "visual information" would indicate that it modulates the oculomotor response. In the second analysis, we normalized the oculomotor response to the change in visual information by collapsing across the different visual information levels to allow for comparisons across groups. With this normalized variable, a value larger than zero corresponds to an effect of visual information. For this second analysis, we used the following factors: age, group, target velocity and trial number (first vs. subsequent trials).

These analyses were conducted using the afex (Singmann et al. 2018), Ime4 (Bates et al. 2015) and ImerTest (Kuznetsova et al. 2017) packages in $R$ (R Core Team 2018). Post-hoc pairwise comparisons were conducted in the form of robust independent and dependent $t$-tests using the WRS2 R package (Mair and Wilcox 2018).

\section{RESULTS}

Out of 117 participants, 21 were excluded based on the inclusion and exclusion criteria. The final sample included 21 amblyopic children ( $M$-age: 9 years; $S D$ : 2 years; $n: 8$ females and 13 males), of which 11 were strabismic, 6 were anisometropic and 4 were organic amblyopes; 20 age-matched control children ( $M$-age: 9 years; SD: 2 years; $n$ : 9 females and 11 males); 18 amblyopic adults ( $M$ - 
age: 33 years; $S D: 17$ years; $n: 8$ females and 10 males), of which 14 were strabismic and 4 were anisometropic amblyopes; and 37 control adults (M-age: 32 years; $S D$ : 11 years; $n: 21$ females and 16 males). The 20 control children all participated in both the noisy and standard versions of the test. The 37 control adults took part in either one version (26 control adults) or both versions of the protocol (11 control adults). This resulted in 23 recordings of control adults with a standard target, and 25 recordings of control adults with a noisy target.

Fig. 2 (a) Overall pursuit performance during Trial \#1, averages, per group, of participant's average eye velocity traces during Training trials at $15 \%$ s and $20 \%$ (top: pediatric, bottom: adult). The surrounding error bars indicates $95 \% \mathrm{Cl}$ (b) Pursuit onset and steady-state pursuit gain, for training trials at $15 \%$ s and $20 \%$ s. Dots correspond to individual participants. Error bars are centered on the average of participant's averages and indicate the $95 \% \mathrm{Cl}$

\section{PURSUIT ONSET AND GAIN ACROSS AGES AND GROUPS}

We compared the effect of age (children vs. adult) and group (controls with standard target, controls with noisy target and amblyopic patients with standard target) on smooth pursuit latency and steadystate pursuit gain (Fig. 2).

For the pursuit onset, we found that children had a longer reaction time than adults (main effect of age: $F(1,86.51)=22.5, p<0.0001$ ) and that group type also influenced this parameter (main effect of group: $\mathrm{F}(2,156.52)=295.8, \mathrm{p}<0.0001)$. More precisely, control participants with a standard target had the shortest reaction time (children: $123 \mathrm{~ms}, \mathrm{Cl}=[119 \mathrm{~ms}, 126 \mathrm{~ms}]$; adults: $103 \mathrm{~ms}, \mathrm{Cl}=[99 \mathrm{~ms}, 107 \mathrm{~ms}]$ ) while the control participants with a noisy target had the longest reaction time (children: $156 \mathrm{~ms}, \mathrm{Cl}=[151 \mathrm{~ms}$, $161 \mathrm{~ms}]$; adults: $143 \mathrm{~ms}, \mathrm{Cl}=[139 \mathrm{~ms}, 147 \mathrm{~ms}])$. Amblyopic patients had a reaction time between the two conditions of the control participants, very close to controls with a standard target (children: $126 \mathrm{~ms}$, $\mathrm{Cl}=[120 \mathrm{~ms}, 131 \mathrm{~ms}]$; adults: $121 \mathrm{~ms}, \mathrm{Cl}=[114 \mathrm{~ms}, 127 \mathrm{~ms}])$. The interaction between age and group was also significant $(F(2,156.52)=3.83, p=0.02)$. Indeed, there was a significant onset difference of $10-20 \mathrm{~ms}$ between children and adults in all conditions except in the amblyopic participants. For adults, controls with a standard target had a shorter onset than amblyopic participants (standard vs. amblyope: Mdiff=$17.41 \mathrm{~ms}, \mathrm{Cl}=[-24.23 \mathrm{~ms},-10.59 \mathrm{~ms}], \mathrm{Yt}=-4.81, \mathrm{p}<0.0001$, effect size $=0.706)$, amblyopic participants had a shorter onset than controls with a noisy target (noisy vs. amblyope: Mdiff $=22.75 \mathrm{~ms}, \mathrm{Cl}=[15.23 \mathrm{~ms}$, $30.27 \mathrm{~ms}$ ], $Y t=6.21, p<0.0001$, effect size $=0.78$ ), and controls with a standard target had a shorter onset than controls with a noisy target (standard vs. noisy: Mdiff $=-40.16 \mathrm{~ms}, \mathrm{Cl}=[-45.66 \mathrm{~ms},-34.65 \mathrm{~ms}], \mathrm{Yt}=-$ $14.55, p<0.0001$, effect size $=0.99$ ). A similar trend was observed in children with the exception of an absence of significant difference between the standard and amblyopic groups. Thus, in children, controls with a standard target had a shorter onset than controls with a noisy target (standard vs. noisy: Mdiff=$33.33 \mathrm{~ms}, \mathrm{Cl}=[-39.23 \mathrm{~ms},-27.43 \mathrm{~ms}], \mathrm{Yt}=-11.3, \mathrm{p}<0.0001$, effect size $=0.83$ ), and amblyopic children had a shorter onset than controls with a noisy target (noisy vs. amblyope: Mdiff $=30.38 \mathrm{~ms}, \mathrm{Cl}=[23.23 \mathrm{~ms}$, $37.53 \mathrm{~ms}], Y t=8.29, p<0.0001$, effect size $=0.92)$. However, the onsets of control children with a standard target and of amblyopic children were not significantly different (standard vs. amblyope: Mdiff $=-2.95 \mathrm{~ms}$, $\mathrm{Cl}=[-9.11 \mathrm{~ms}, 3.21 \mathrm{~ms}], \mathrm{Yt}=-0.9, \mathrm{p}=0.34$, effect size $=0.16)$.

The quality of the pursuit, as measured by the pursuit gain, was lower in children than in adults $(F(1,90.03)=2.7, p=0.1)$ but was clearly influenced by the group (main effect: $F(2,153.89)=39.73$, $p<0.0001)$ and, as expected, by target velocity $(F(1,150.05)=198.7, p<0.0001)$. Interestingly, the effect of group on the pursuit gain was different for the two age categories (interaction between age and group: $\mathrm{F}(2,153.89)=3.94, \mathrm{p}=0.02)$. In controls with a standard or noisy target, pursuit gain of children (standard: mean $=0.75, \mathrm{Cl}=[0.71,0.79]$; noisy: mean $=0.66, \mathrm{Cl}=[0.61,0.71]$ ) was lower than in adults (standard: mean $=0.87, \mathrm{Cl}=[0.83,0.91]$; $\mathrm{Mdiff}=-0.12 \mathrm{Cl}=[-0.17,-0.06], \mathrm{Yt}=-4-41, \mathrm{p}<0.0001$, effect size=0.67; noisy: mean=0.75, $\mathrm{Cl}=[0.69,0.80]$; Mdiff=-0.09 $\mathrm{Cl}=[-0.16,-0.02], \mathrm{Yt}=-2.44, \mathrm{p}=0.02$, effect size $=0.37$ ). However, in amblyopic patients, the pursuit gains were not significantly different between children and adults (children: mean $=0.75, \mathrm{Cl}=[0.68,0.82]$ ) and adults: mean $=0.71, \mathrm{Cl}=[0.62,0.80]$; Mdiff $=0.04, \mathrm{Cl}=[-0.06,0.15], \mathrm{Yt}=0.77, \mathrm{p}=0.43$, effect size $=0.14)$. Control children with a noisy target had a significantly lower gain than control children with a standard target (standard vs. noisy: 
Mdiff=0.09, $\mathrm{Cl}=[0.03,0.15], \mathrm{Yt}=3.05, \mathrm{p}=0.005$, effect size=0.49) or than amblyopic children (noisy vs. amblyope: Mdiff=-0.09, $\mathrm{Cl}=[-0.17,-0.01], \mathrm{Yt}=-2.15, \mathrm{p}=0.004$, effect size=0.34). However, the gains of control children with a standard target and of amblyopic children were not significantly different (standard vs. amblyope: Mdiff $=0.004, \mathrm{Cl}=[-0.07,0.08], \mathrm{Yt}=0.11, \mathrm{p}=0.91$, effect size $=0.04$ ). This trend across groups was different for adults. Amblyopic adults had a lower gain than control adults with a standard target (standard vs. amblyope: $\mathrm{Mdiff}=0.16, \mathrm{Cl}=[0.07,0.25], \mathrm{Yt}=3.47, \mathrm{p}=0.004$, effect size $=0.62$ ), and control adults with a noisy target also had a lower gain than control adults with a standard target (standard vs. noisy: $M d i f f=0.12, \mathrm{Cl}=[0.06,0.19], \mathrm{Yt}=3.71, \mathrm{p}<0.0001$, effect size $=0.50$ ). There was no significant difference between the amblyopic and noisy groups (noisy vs. amblyope: Mdiff $=0.04, \mathrm{Cl}=[-0.06,0.14], \mathrm{Yt}=0.82, \mathrm{p}=0.41$, effect size $=0.12$ ).

Fig. 3 Effect of prior and visual information on eye velocity in children (a) Averages of participant's average eye velocity traces during training and test trials with the same target velocity (first row: 15 $\mathrm{deg} / \mathrm{s}$; second row: $20 \mathrm{deg} / \mathrm{s}$ ), but different priors of target velocity for trials \#2-\#4. blue traces correspond to training trials, red/green traces to test trials. Error bars indicate $95 \% \mathrm{Cl}$ (b) Averages of participant's average eye velocity traces during training and test trials with the same priors of target velocity (first row: $15 \mathrm{deg} / \mathrm{s}$; second row: $20 \mathrm{deg} / \mathrm{s}$ ), but different target velocity, for trials \#2-\#4. Blue traces correspond to training trials, red/green traces are test trials. Error bars indicate $95 \% \mathrm{Cl}$

\section{EFFECT OF PRIOR AND VISUAL INFORMATION ON EYE VELOCITY}

\section{PRIOR}

As we have previously shown (Deravet et al. 2019) that prior information has a larger influence when the target is noisy than when it is standard, we hypothesized that the effect of prior information would be higher in the trials with a noisy target for control participants and in the trials of amblyopic patients, as a result of the deterioration of the quality of visual information. As illustrated in Fig. 3a (first row, red trace), the effect of prior visual information was assessed by first displaying a target at a velocity of 20 $\mathrm{deg} / \mathrm{s}$ during the training trials; during the consecutive test trial, with a target velocity of $15 \mathrm{deg} / \mathrm{s}$, the participant, influenced by his short-term memory of visual information, did not follow the target at 15 $\mathrm{deg} / \mathrm{s}$ but at a higher velocity. Similarly, in the blocks in which the target velocity in the training trials was lower than in the test trial (second row, green trace), after having followed a target at a velocity of 15 $\mathrm{deg} / \mathrm{s}$ during the training trials, the participant, faced with a target at a velocity of $20 \mathrm{deg} / \mathrm{s}$ during the test trial, did not follow the target at $20 \mathrm{deg} / \mathrm{s}$ but at a lower velocity. This suggests that the target velocity from previous trials influences the smooth pursuit response in subsequent trials.

Fig. 4 Normalized effect of prior and visual information on eye velocity and saccades in children compared to adults. Normalized effect of prior (a) and visual (b) information, on eye velocity, with participant's averages as dots, and error bars corresponding to the standard error of the mean. Normalized effect of prior (c) and visual (d) information on saccade amplitude. Dots are individual averages of normalized residuals, and error bars correspond to the standard error of the mean. Participant's averages in (a) and (c) are based on trials \#2 and following, while in (b) and (d), they are based on trials \#1 and following.

For comparison of the effect of prior across ages and groups, we normalized the smooth pursuit data such that we could get one normalized effect of prior target velocity independently from the fact that it was linked to a higher or lower target velocity (see methods and Deravet et al. 2018). The normalized effect of prior on eye velocity across ages and groups is shown in Fig. 4a. We found that prior information had a significant effect on normalized eye velocity (main effect of presence of prior information: $F(1,395.05)=34.59, p<0.0001)$ and that it was slightly modulated by target velocity (interaction between prior and target velocity: $F(1,395.05)=4.21, p=0.04)$ with the effect of the prior being slightly larger for higher target velocity (mean and $\mathrm{Cl}$ of normalized effect at $15 \mathrm{deg} / \mathrm{s}: 0.12$, $[0.08,0.16], 20 \mathrm{deg} / \mathrm{s}: 0.23,[0.19,0.27])$. However, we did not find any evidence for a difference across groups (interaction between prior and group: $\mathrm{F}(2,395.06)=0.95, \mathrm{p}=0.39$ ).

\section{VISUAL}


Based on our previous study (Deravet et al. 2019), we predicted that the effect of visual information would be higher in controls with a standard target, compared to controls with a noisy target or the amblyopic participants. As illustrated in Fig. 3b (first row), after participants have followed the target at $15 \mathrm{deg} / \mathrm{s}$ in test trials, they are faced with a target at a higher (green trace), similar (blue trace) or lower velocity (red trace) during test trial. Eye velocity is thus expected to adapt to the new target velocity.

We found that visual information had a significant effect on eye velocity (main effect of presence of visual information: $F(2,634.01)=431.6, p<0.0001)$. The effect of visual information on eye velocity was also expected to differ depending on target velocity. Indeed, we found that smooth pursuit steady-state gain was higher when target velocity was higher (main effect of target velocity: $F(2,634.01)=429.48, p$ $<.0001$ ). That is, increasing or decreasing target velocity leads to an increase or decrease in smooth eye velocity. To analyze this further, we normalized these data in such a way that we could pool the data for the increase and decrease in target velocity together, yielding a normalized effect of visual information for each participant and each condition. The normalized effect of visual information on eye velocity is shown on Fig. 4b. The effect differed across groups (main effect: $F(2,193.48)=24.15$, $\mathrm{p}<0.0001)$. As expected, the normalized effect of visual information was larger for the control participants with the standard target than those with the noisy target (standard vs. noisy: Mdiff $=0.19$ $\mathrm{Cl}=[0.14,0.24], \mathrm{Yt}=7.748, \mathrm{p}<0.0001$ ) or for the amblyopes (standard vs. amblyope: $\mathrm{Mdiff}=0.12$ $\mathrm{Cl}=[0.07,0.19], \mathrm{Yt}=4.151, \mathrm{p}<0.0001)$. In other words, a clear vision of the target allows one to better tune her/his eye velocity to target velocity.

Furthermore, this modulation was different across the two age groups (interaction between group and age: $F(2,193.48)=15.76, p<0.0001)$. The effect of visual information was larger in adults than in children for control participants with a standard target (child vs. adult: Mdiff $=-0.15, \mathrm{Cl}=[-0.22,-0.07]$, $\mathrm{Yt}=-3.96, \mathrm{p}=0.001$, effect size $=0.48$ ) but was lower for control adults with the noisy target (child vs. adult: Mdiff $=0.08, \mathrm{Cl}=[0.007,0.15], \mathrm{Yt}=2.3, \mathrm{p}=0.03$, effect size $=0.25)$ and for the amblyopic adults with the standard target (child vs. adult: $\mathrm{Mdiff}=0.13, \mathrm{Cl}=[0.04,0.23], \mathrm{Yt}=2.80, \mathrm{p}=0.006$, effect size $=0.33$ ) (Fig. 4b). In other words, the modulation of the effect of visual information on the smooth pursuit response by group was more limited in children (standard vs. noisy: effect size $=0.22$; standard vs. amblyope: effect size $=0.03$ ) than in adults (standard vs. noisy: effect size $=0.77$; standard vs. amblyope: effect size $=0.70$ ).

\section{EFFECT OF PRIOR AND VISUAL INFORMATION ON SACCADES}

\section{PRIOR}

As we found that prior and visual information also influenced the amplitude of catch-up saccades in our previous work (Deravet et al. 2019), we performed the same analysis on the saccadic amplitude (Fig. 4c and d). As for the pursuit gain, we analyzed the normalized effect of prior and visual information (see methods and Deravet et al. 2018). We found that prior information influenced the normalized saccadic amplitude of the first catch-up saccade: (main effect of prior information: $F(1,395.85)=16.81, p<0.0001)$. That is, saccade amplitude was larger (resp. lower) when the target velocity of the previous trials was higher (resp. lower) than in the current trial. Similarly to the pursuit gain results, we did not find any evidence that group or age influenced the effect of prior information on the normalized saccade amplitude (interaction between prior and group: $F(2,395.8)=1.54, p=0.22$; interaction between prior and age: $F(1,395.85=2.96, p=0.09)$.

\section{VISUAL}

Visual information was also able to modulate saccadic amplitude (main effect of visual information on saccade amplitude: $F(2,634.02)=345.16, p<.0001$ ), which simply shows that saccade amplitude is larger when target velocity is larger. We analyzed this further with the normalized saccadic amplitude in order to test for differences across groups. Participant group modulated the effect of visual information on normalized saccadic amplitude (main effect: $F(2,221.49)=4.46, p=0.01$ ). As expected, 
the effect of visual information on saccadic amplitude was larger for the control participants with a standard target than for those with a noisy target (standard vs. noisy: Mdiff $=0.18, \mathrm{Cl}=[0.08,0.28]$, $Y \mathrm{t}=3.84, \mathrm{p}<0.0001$ ) and larger for the amblyopic patients than for the controls with a noisy target (noisy vs. amblyope: $\mathrm{Mdiff}=-0.22, \mathrm{Cl}=[-0.35,-0.1], \mathrm{Yt}=-3.65, \mathrm{p}<0.0001)$. In contrast to what was observed for smooth pursuit, we did not find any evidence that the effect of visual information on saccade amplitude was different for children and adults (interaction between visual information and age: $F(1,401.38)=0.01, p=0.93)$.

\section{DISCUSSION}

This study first showed that the pursuit onset was delayed and the gain was lower in control children with a noisy target in comparison to amblyopic children or control children with a standard target. Unexpectedly, the pursuit onset and gain were not different between control children with a standard target and amblyopic children. The comparison between pediatric and adult pursuit characteristics showed that, while the pursuit performance of control children with a standard or noisy target improved with age, with higher gains and shorter latencies in control adults with these targets, it did not improve with age in amblyopic participants. In addition, the comparison also showed that the variation of target velocity had a significant effect on pursuit onset and on gain, in both children and adults. Secondly, a significant effect of prior and of visual information on pursuit velocity and on saccades was found across all participants. Moreover, the modulation of the effect of visual information on the pursuit velocity by group was more limited in children.

The modeling of brain decisions based on multiple sensory inputs (e.g., visual, auditory, somatosensory) has been achieved by several authors (Ernst and Banks 2002; Jacobs and Fine 1999; Su et al. 2017) using Bayesian integration, by summing the inputs with a weight based on the respective reliability of each input. This modeling may explain that amblyopic children perform like control children in motor tasks involving manual dexterity, balance or aiming skills (Hemptinne et al. 2020). Despite their less reliable visual input, with an altered stereopsis, amblyopic children probably rely to a greater extent on other sensory inputs, such as vestibular or somatosensory information, in balance tasks, and on other depth cues, such as light/shadow and perspective, in aiming tasks. The present study has brought additional insights on the characteristics of the neural decision model of amblyopic participants in visual tasks. Orban de Xivry et al. showed in 2013 that a memory of past experience was integrated with sensory inputs with a reliability-based approach using Kalman filtering to allow motor decision. The pursuit performance deficits in amblyopic adults, reflected by a lack of gain and latency improvement in amblyopic participants, as opposed to controls, suggest a smaller weight associated with visual input in amblyopic adults, compared to control adults. This confirms the findings of Deravet et al. (2019).

Amblyopic children achieved a better pursuit performance, with a higher gain and a shorter latency, compared to control children tested with a stimulus assumed to mimick the blurriness perceived by amblyopic children. Thus, with a similarly deteriorated visual input, amblyopic children better adapted their pursuit than control children. This may derive from a possible unfair comparison between visual conditions, but also from compensation mechanisms developed in the long run by amblyopic children. As the degraded visual information is present at an early age in amblyopic children, there seems to be a built strategy with a greater tolerance for blurriness at some point of their visual development, while control children have no time to adapt to an immediate degradation of the visual information.

Furthermore, the presence of amblyopia prevents future progress and maturation. Beyond a given point, control children seem to pursue their visual maturation, while amblyopic children may no longer make visual progress. Therefore, control adults perform better than amblyopic adults.

The continuous maturation of the pursuit system until late adolescence explains the improvement with age of pursuit latency and gain in control participants (Rütsche et al. 2006; Salman, Sharpe, Lillakas, et al. 2006; Von Hofsten and Rosander 1997). In amblyopic children, however, the present study 
suggests a blockage of the development of the pursuit system, at the origin of the already described deficits in motion sensitivity in amblyopes (Kiorpes et al. 2006). Our results suggest that the motion processing deficits in amblyopic adults result from the absence of maturation of the pursuit system. While in childhood, it seems that the built strategy of amblyopia is sufficient to ensure a good pursuit performance, the performance becomes poor in adulthood. Thus, it is likely that amblyopic adults will rely on the visual input associated with a moving target to a smaller extent than control adults. A functional magnetic resonance imaging analysis of amblyopic patients showed a topographic disorganization in all early visual areas (Clavagnier et al. 2015). In line with our study, these researchers depicted the amblyopic visual system as an immature system with a lower spatial resolution and a disordered topographical map. The development of the oculomotor system in amblyopic patients contrasts with other deficits, such as cerebral palsy, in which children's oculomotor system matures as fast as or even faster than in control children, thereby compensating for impaired motor function (Ego et al. 2015). The different neural development mechanisms induced by amblyopia as opposed to cerebral palsy may reflect different plasticity mechanisms in the sensory as opposed to the motor system.

While visual information had a significantly different effect on eye velocity and saccades for amblyopic and control patients with a noisy or standard target, the effect of prior information on these two parameters was not significantly different between these three groups. It could have been expected that amblyopic patients, compared to controls, would have given more weight to prior information to compensate for the lower reliability of their visual perception. However, in binocular viewing conditions, amblyopic patients can rely on the visual input of their dominant eye. The pathways involved in the integration of prior information mature in the long run based on binocular information, and notably the input of the dominant eye. Therefore, these pathways are expected to develop and to be integrated with a similar weight in amblyopic patients, because of the inhibition of visual input from the amblyopic eye by the dominant eye. In the present study, while visual information was captured only by the unpatched eye, prior information might have been processed by pathways developed in binocular conditions, with the input of the dominant eye. Consequently, the comparable effect of prior information in the amblyopic and control patients might require a binocular processing of prior information, after visual information is gathered by both retinas. This hypothesis is consistent with the suggestion that this different processing of visual and prior motion information is probably performed in distinct cortical areas, the MT area (Osborne et al. 2007) and the frontal eye fields (Darlington et al. 2017) respectively.

Regarding the between-group difference in the effect of visual information on eye velocity, a larger effect of visual information was observed in control adults with a standard target compared to control children. However, with a blurry target, children showed a larger effect of visual information either in control participants with a noisy target or in amblyopic participants with a standard target. Thus, adults, faced with high quality visual information, adapted their oculomotor behavior to a larger extent than children, but with low quality visual input, adults decreased the weight given to visual information. These findings might be explained by an oculomotor control in adults trained to low tolerance for errors. In children, to the contrary, tolerance for errors might be higher, leading to a more important weight given to visual information despite its low quality. This oculomotor behavior in children may be related to the findings of Ego et al., 2013, who showed that despite their lower pursuit performance, children did not compensate with more catch-up saccades. To the contrary, children performed less saccades than adults and saccade latencies were longer for children. These observations, while children are able to perform saccades with latencies as short as adults, were explained by a larger tolerance for average position errors in children. This larger tolerance in children may suggest a more approximate adaptation of eye velocity to target velocity, with a more limited influence of prior target velocity on saccades amplitude after the target velocity changes.

While the modulation by group of the effect of visual information on pursuit velocity was different between adults and children, there was no evidence that the effect of visual information on saccade 
amplitude was different between children and adults. This may be explained by the different timeframes of development for pursuit and saccades. Whereas the saccadic system completes its maturation at about 6 years (Ego et al. 2013), the pursuit system keeps developing until late adolescence. As most tested children were older than 6 years old, we could expect that their control of saccades based on visual information would not differ significantly from adults, as opposed to their tuning of pursuit velocity.

\section{REFERENCES}

Accardo, A. P., Pensiero, S., Da Pozzo, S., \& Perissutti, P. (1995). Characteristics of horizontal smooth pursuit eye movements to sinusoidal stimulation in children of primary school age. Vision Research, 35, 539-548. https://doi.org/10.1016/0042-6989(94)00145-C

Bates, D., Mächler, M., Bolker, B. M., \& Walker, S. C. (2015). Fitting linear mixed-effects models using Ime4. Journal of Statistical Software, 67, 1-48. https://doi.org/10.18637/jss.v067.i01

Becker, W., \& Jürgens, R. (1979). An analysis of the saccadic system by means of double step stimuli. Vision Research, 19(9), 967-983. https://doi.org/10.1016/0042-6989(79)90222-0

Clavagnier, S., Dumoulin, S. O., \& Hess, R. F. (2015). Is the cortical deficit in amblyopia due to reduced cortical magnification, loss of neural resolution, or neural disorganization? Journal of Neuroscience, 35, 14740-14755. https://doi.org/10.1523/JNEUROSCI.1101-15.2015

Darlington, T. R., Tokiyama, S., \& Lisberger, S. G. (2017). Control of the strength of visual-motor transmission as the mechanism of rapid adaptation of priors for Bayesian inference in smooth pursuit eye movements. Journal of Neurophysiology, 118, 1173-1189. https://doi.org/10.1152/jn.00282.2017

Deravet, N., Blohm, G., de Xivry, J. J. O., \& Lefèvre, P. (2018). Weighted integration of short-term memory and sensory signals in the oculomotor system. Journal of Vision, 18(5), 1-19. https://doi.org/10.1167/18.5.16

Deravet, N., Yüksel, D., Orban de Xivry, J.-J., \& Lefèvre, P. (2019). Integration of past and current visual information during eye movements in amblyopia. In Progress in Brain Research (pp. 4563). https://doi.org/10.1016/bs.pbr.2019.04.034

Ego, C., de Xivry, J. J. O., Nassogne, M. C., Yüksel, D., \& Lefèvre, P. (2013). The saccadic system does not compensate for the immaturity of the smooth pursuit system during visual tracking in children. Journal of Neurophysiology, 110, 358-367. https://doi.org/10.1152/jn.00981.2012

Ego, C., Orban de Xivry, J. J., Nassogne, M. C., Yüksel, D., \& Lefèvre, P. (2015). Spontaneous improvement in oculomotor function of children with cerebral palsy. Research in Developmental Disabilities. https://doi.org/10.1016/j.ridd.2014.10.025

Ernst, M. O., \& Banks, M. S. (2002). Humans integrate visual and haptic information in a statistically optimal fashion. Nature, 415(6870), 429-33. https://doi.org/10.1038/415429a

Fukushima, J., Hatta, T., \& Fukushima, K. (2000). Development of voluntary control of saccadic eye movements: I. Age- related changes in normal children. Brain and Development, 22, 173-180. https://doi.org/10.1016/S0387-7604(00)00101-7

Hemptinne, C., Aerts, F., Pellissier, T., Ramirez Ruiz, C., Alves Cardoso, V., Vanderveken, C., \& Yüksel, D. (2020). Motor skills in children with strabismus. Journal of AAPOS, S1091-8531(20), 30055-0. https://doi.org/10.1016/j.jaapos.2020.01.005

Irving, E. L., Steinbach, M. J., Lillakas, L., Babu, R. J., \& Hutchings, N. (2006). Horizontal saccade dynamics across the human life span. Investigative Ophthalmology and Visual Science, 47, 2478-2484. https://doi.org/10.1167/iovs.05-1311

Jacobs, R. A., \& Fine, I. (1999). Experience-dependent integration of texture and motion cues to 
depth. Vision Research, 39(24), 4062-75. https://doi.org/10.1016/S0042-6989(99)00120-0

Katsanis, J., lacono, W. G., \& Harris, M. (1998). Development of oculomotor functioning in preadolescence, adolescence, and adulthood. Psychophysiology, 35, 64-72. https://doi.org/10.1017/S0048577298961406

Kiorpes, L., Tang, C., \& Movshon, J. A. (2006). Sensitivity to visual motion in amblyopic macaque monkeys. Visual Neuroscience, 23(2), 247-256. https://doi.org/10.1017/S0952523806232097

Kuznetsova, A., Brockhoff, P. B., \& Christensen, R. H. B. (2017). ImerTest Package: Tests in Linear Mixed Effects Models. Journal of Statistical Software, 82(13), 1-26. https://doi.org/10.18637/jss.v082.113

Luna, B., Garver, K. E., Urban, T. A., Lazar, N. A., \& Sweeney, J. A. (2004). Maturation of cognitive processes from late childhood to adulthood. Child Development, 75, 1357-1372. https://doi.org/10.1111/j.1467-8624.2004.00745.x

Mair, P., \& Wilcox, R. (2018). WRS2: Wilcox Robust Estimation and Testing. https://cran.rproject.org/web/packages/WRS2/citation.html

Orban de Xivry, J.-J., Coppe, S., Blohm, G., \& Lefèvre, P. (2013). Kalman filtering naturally accounts for visually guided and predictive smooth pursuit dynamics. Journal of Neuroscience, 33, 1730117313. https://doi.org/10.1523/JNEUROSCI.2321-13.2013

Orban De Xivry, J. J., \& Lefèvre, P. (2007). Saccades and pursuit: Two outcomes of a single sensorimotor process. Journal of Physiology, 584, 11-23. https://doi.org/10.1113/jphysiol.2007.139881

Osborne, L. C., Hohl, S. S., Bialek, W., \& Lisberger, S. G. (2007). Time course of precision in smoothpursuit eye movements of monkeys. Journal of Neuroscience, 27, 2987-2998. https://doi.org/10.1523/JNEUROSCI.5072-06.2007

R Core Team, . (2018). R: A language and environment for statistical computing. R Foundation for Statistical Computing, Vienna, Austria. https://www.r-project.org/

Rösblad, B. (1996). Roles of visual information for control of reaching movements in children. Journal of Motor Behavior, 29, 174-182. https://doi.org/10.1080/00222899709600831

Ross, R. G., Radant, A. D., \& Hommer, D. W. (1993). A Developmental Study of Smooth Pursuit Eye Movements in Normal Children from 7 to 15 Years of Age. Journal of the American Academy of Child and Adolescent Psychiatry, 32, 783-791. https://doi.org/10.1097/00004583-19930700000012

Rütsche, A., Baumann, A., Jiang, X., \& Mojon, D. S. (2006). Development of visual pursuit in the first 6 years of life. Graefe's Archive for Clinical and Experimental Ophthalmology, 244, 1406-1411. https://doi.org/10.1007/s00417-005-0248-4

Salman, M. S., Sharpe, J. A., Eizenman, M., Lillakas, L., Westall, C., To, T., et al. (2006). Saccades in children. Vision Research, 46, 1432-1439. https://doi.org/10.1016/j.visres.2005.06.011

Salman, M. S., Sharpe, J. A., Lillakas, L., Dennis, M., \& Steinbach, M. J. (2006). Smooth pursuit eye movements in children. Experimental Brain Research, 169, 139-143. https://doi.org/10.1007/s00221-005-0292-7

Singmann, H., Bolker, B., Westfall, J., \& Aust, F. (2018). a fex: Analysis of Factorial Experiments. $R$ Package Version 0.23-0. https://cran.r-project.org/package1/4afex.

Su, C. C., Cormack, L. K., \& Bovik, A. C. (2017). Bayesian depth estimation from monocular natural images. Journal of Vision, 17(5), 22. https://doi.org/10.1167/17.5.22

Von Hofsten, C., \& Rosander, K. (1997). Development of smooth pursuit tracking in young infants. Vision Research, 37, 1799-1810. https://doi.org/10.1016/S0042-6989(96)00332-X 


\section{1a Example of a stimulus block}

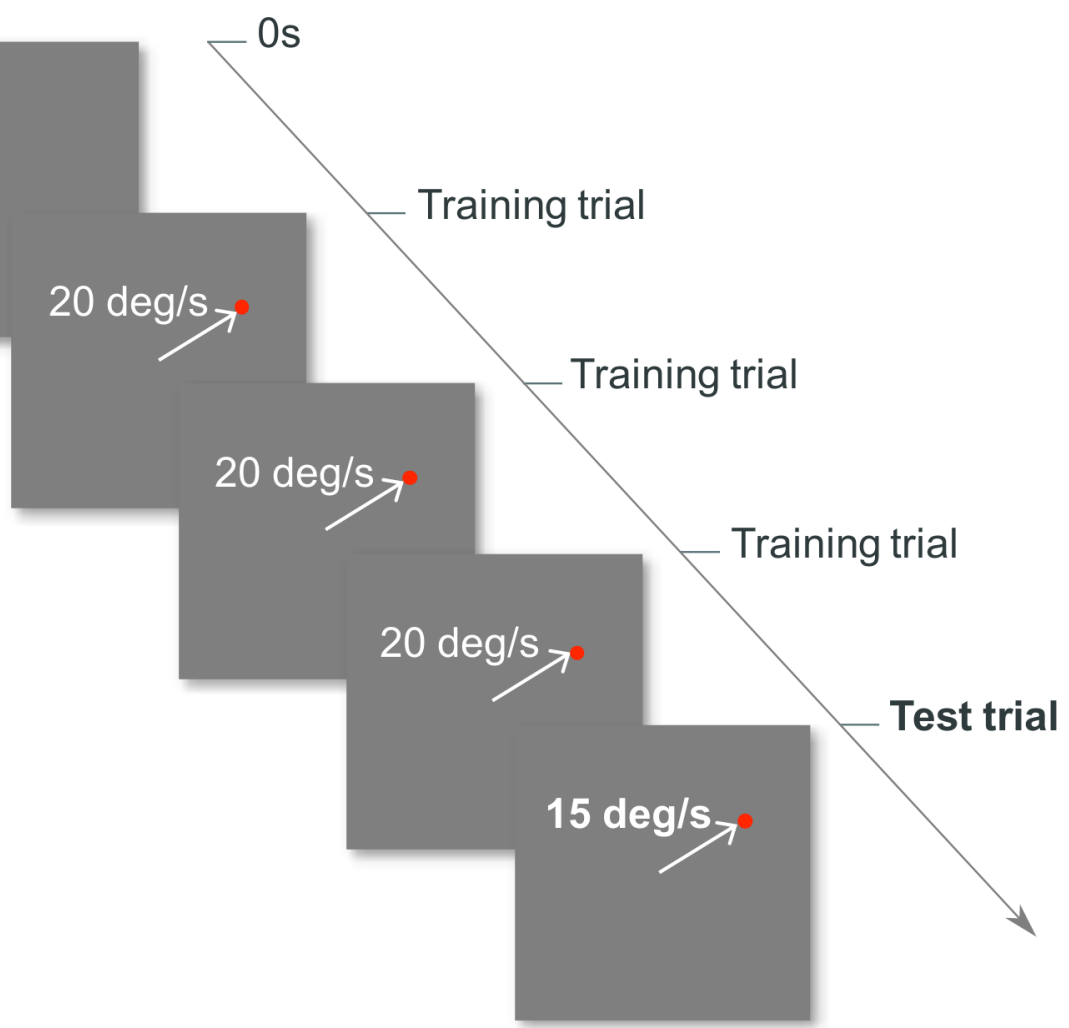

\section{1b Block structure}

$=$


- Standard

- Noisy

- Amblyopic

2a Pursuit during trial \#1 for training trials

Target velocity at $15 \mathrm{deg} / \mathrm{s}$

Target velocity at $20 \mathrm{deg} / \mathrm{s}$

Children
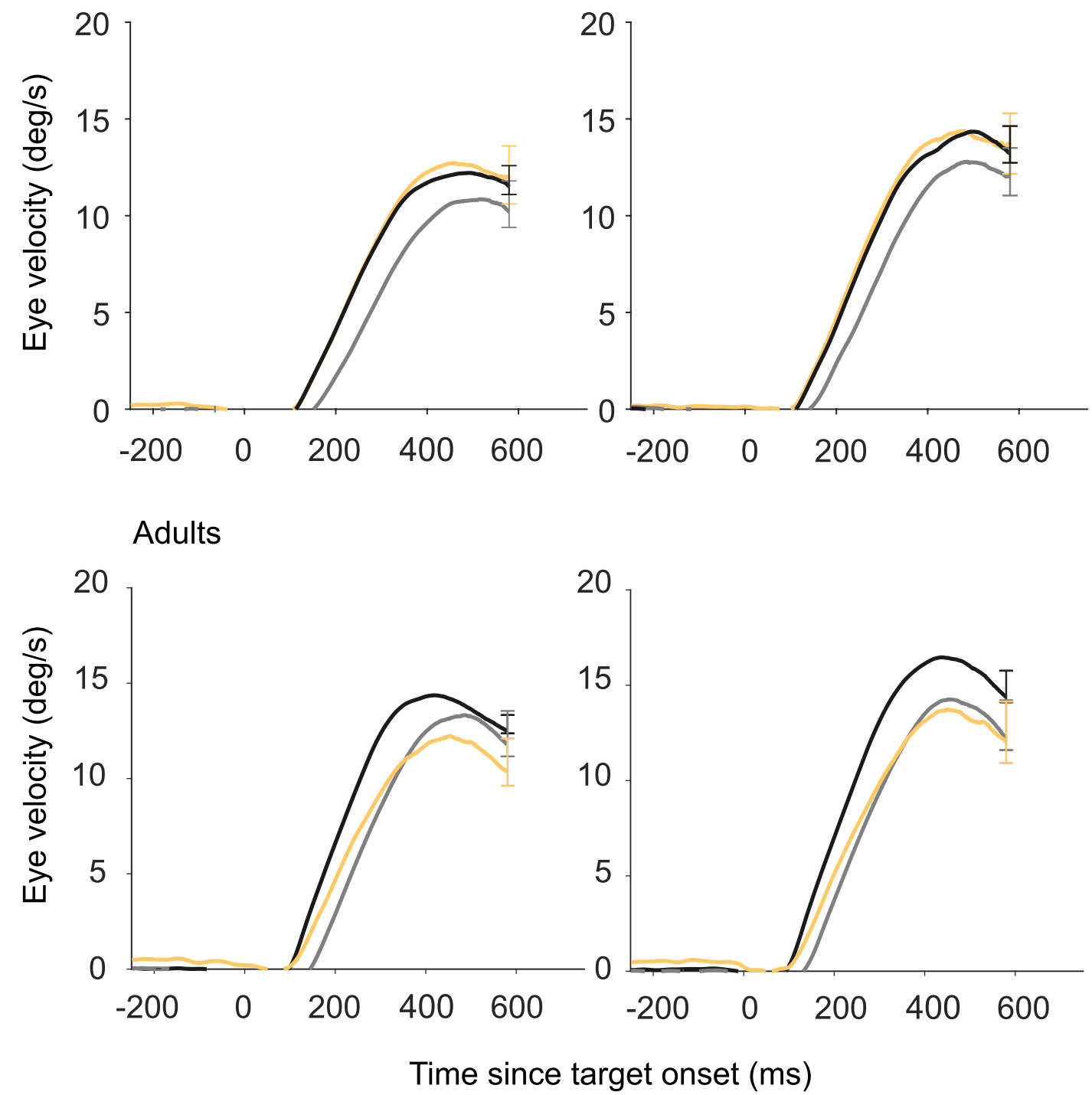

- Children

o Adults

2b Pursuit onset and gain for training trials

Target velocity at $15 \mathrm{deg} / \mathrm{s}$
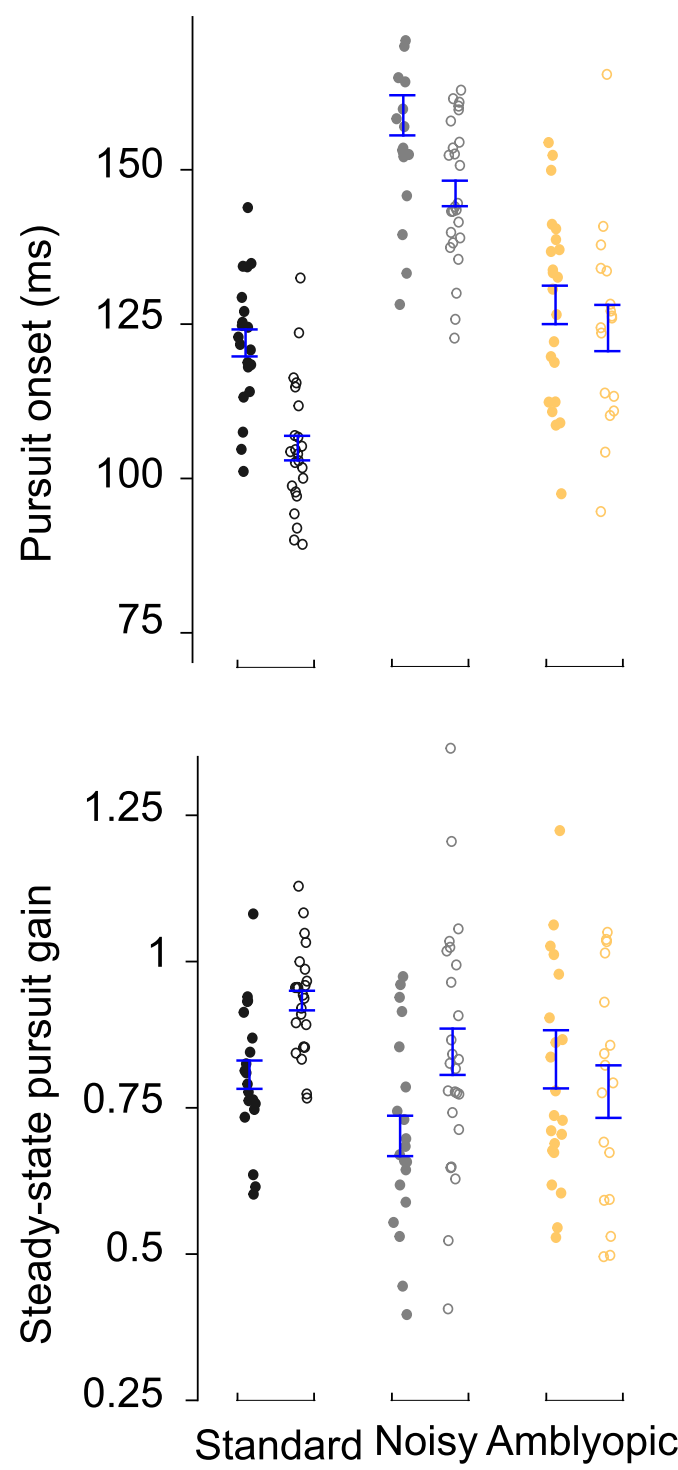

Target velocity at $20 \mathrm{deg} / \mathrm{s}$

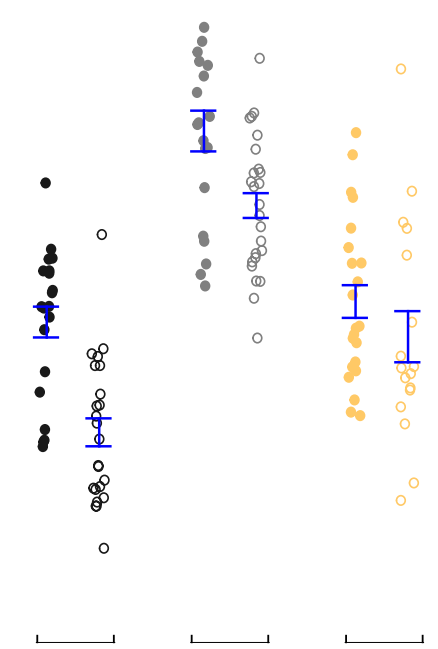




\section{3b Effect of VISUAL on eye velocity in children}

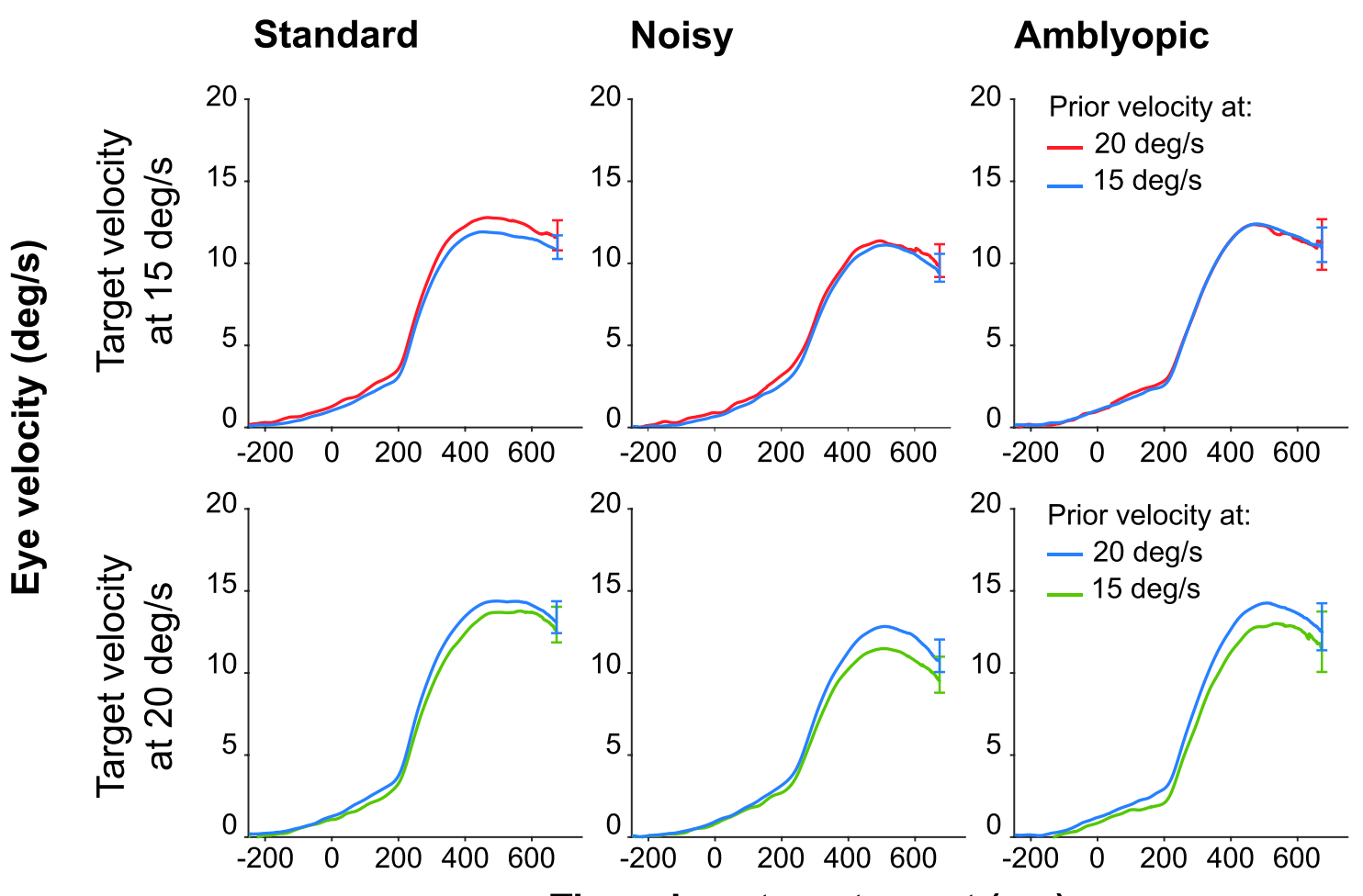
Time since target onset (ms)
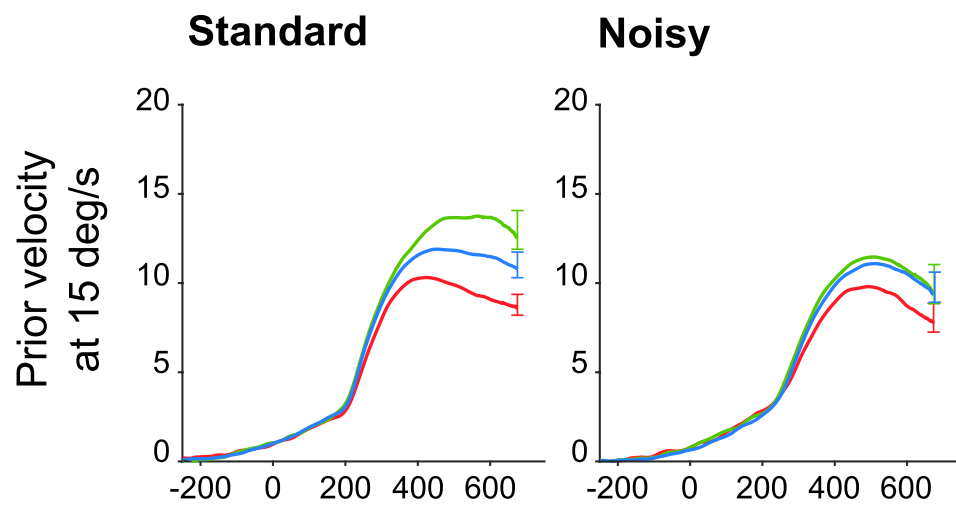

Amblyopic
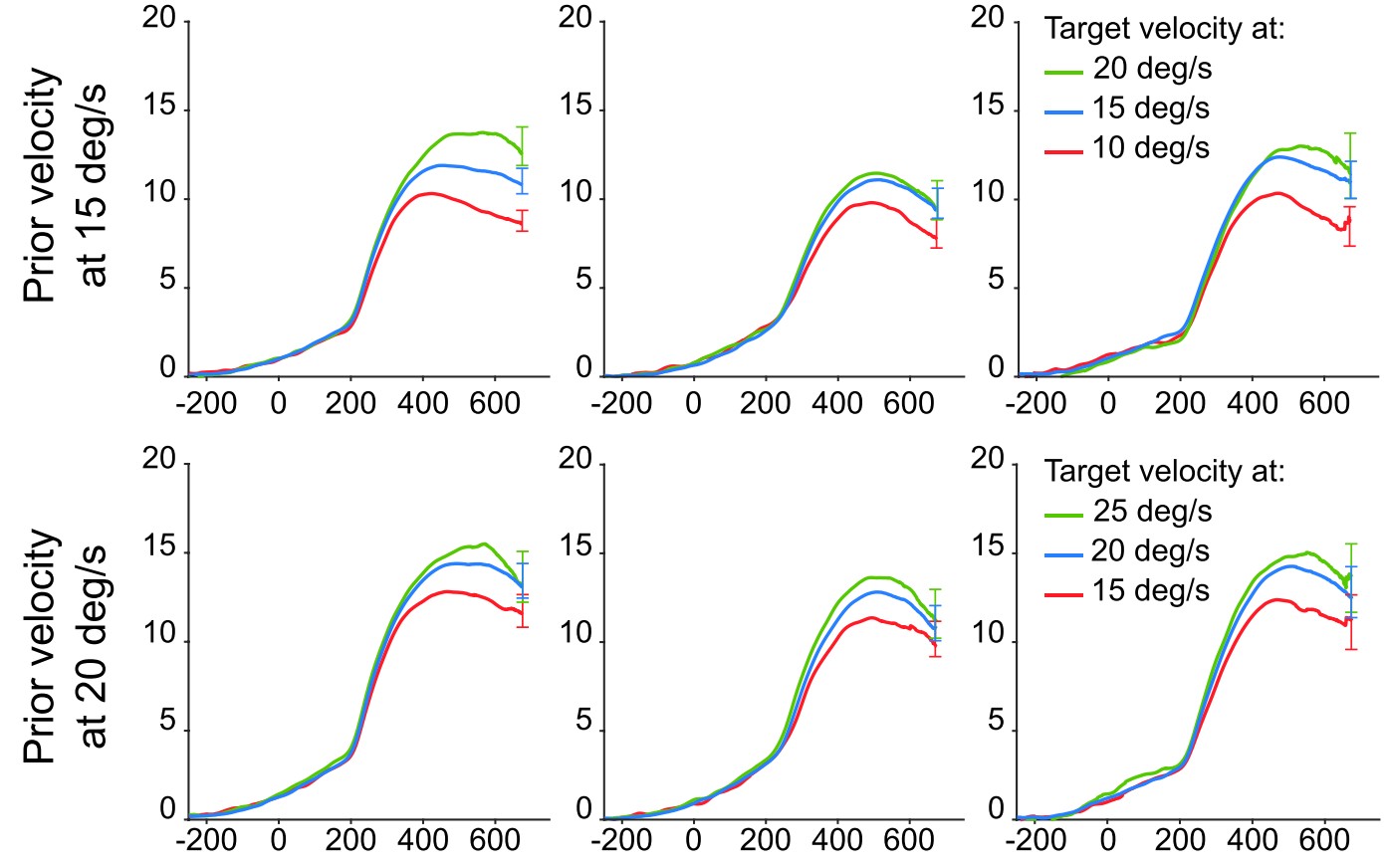

Time since target onset (ms)

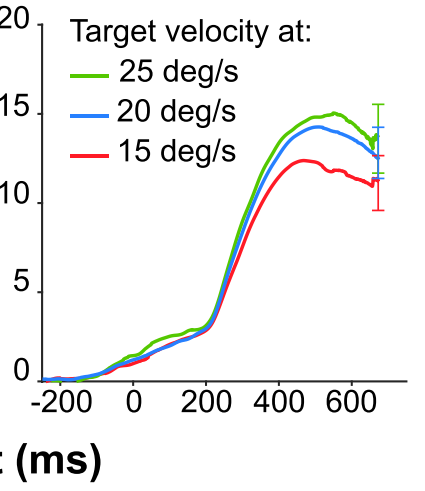


Normalized effect of PRIOR
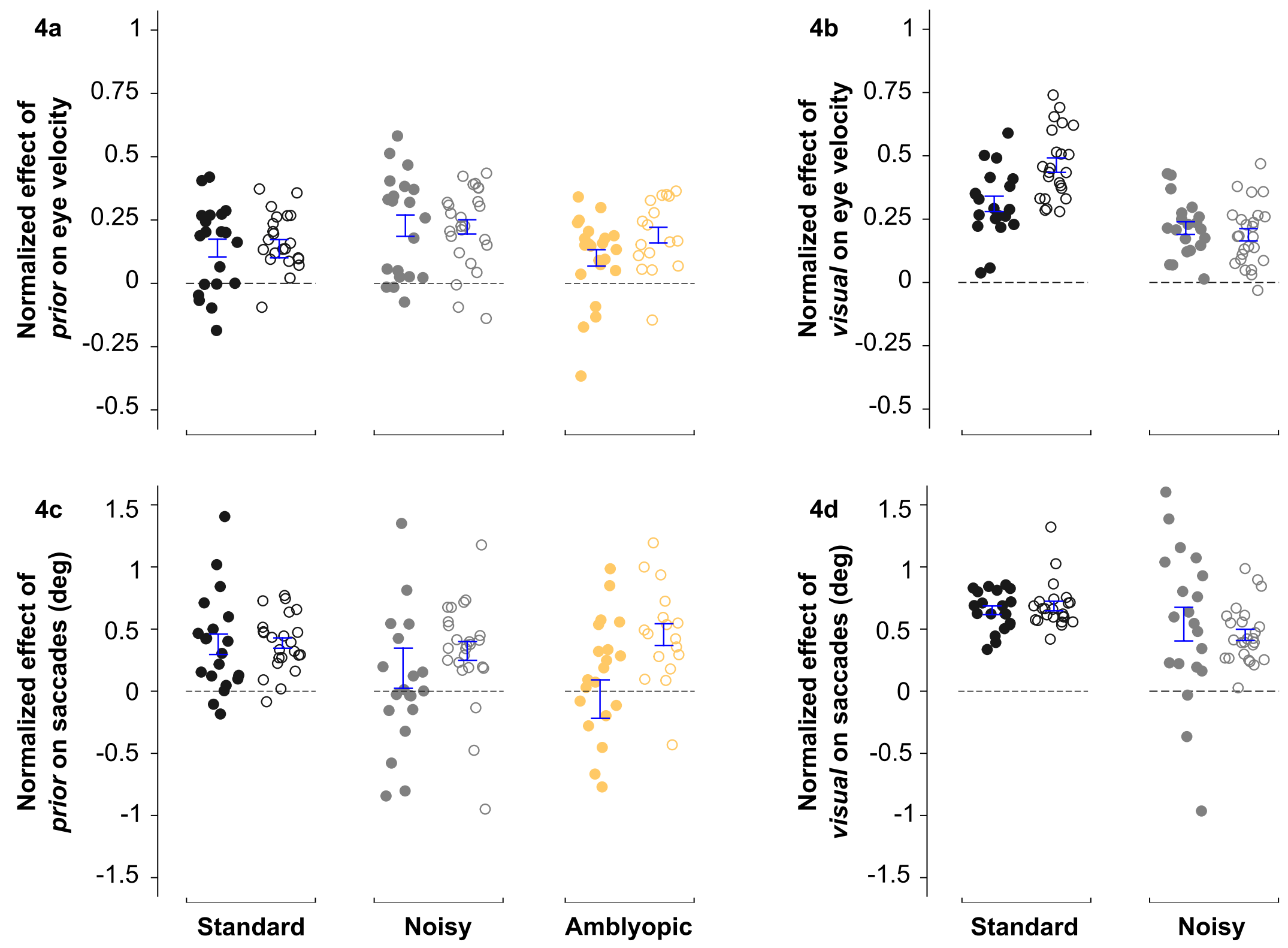

- Standard

- Noisy

- Amblyopic

Normalized effect of VISUAL
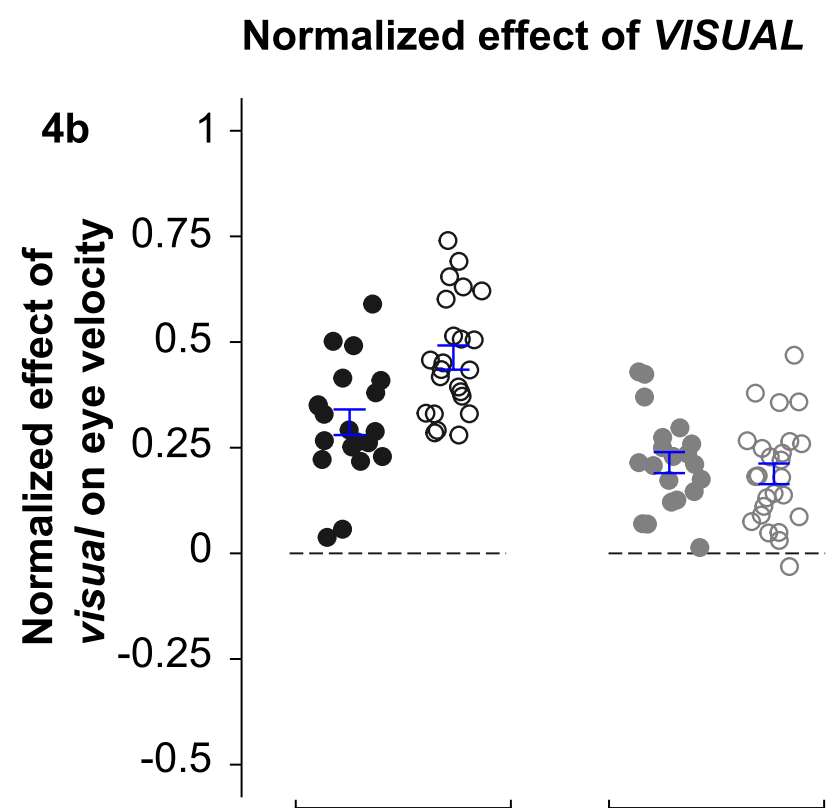

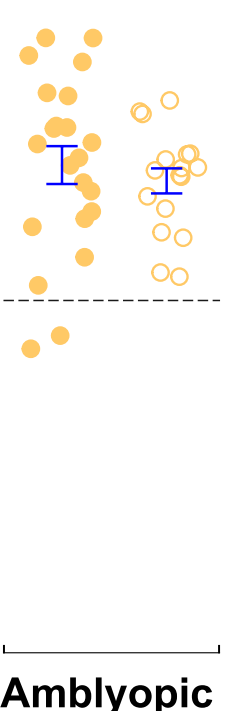

